\title{
Stra8 may inhibit apoptosis during mouse spermatogenesis via the AKT signaling pathway
}

\author{
XUEYI SHEN ${ }^{1,2^{*}}$, CHANGMIN NIU $^{1,2^{*}}$, JIAQIAN GUO ${ }^{1,2^{*}}$, MENGMENG XIA $^{1,2}$, \\ JING XIA ${ }^{1,2}$, YANQIU HU ${ }^{3}$ and YING ZHENG ${ }^{1,2}$ \\ ${ }^{1}$ Department of Histology and Embryology, School of Medicine; ${ }^{2}$ Jiangsu Key Laboratory of \\ Experimental and Translational Non-coding RNA Research; ${ }^{3}$ Center of Reproductive Medicine, \\ Clinical Medical College, Yangzhou University, Yangzhou, Jiangsu 225001, P.R. China
}

Received January 12, 2018; Accepted August 2, 2018

DOI: $10.3892 / \mathrm{ijmm} .2018 .3825$

\begin{abstract}
Stimulated by retinoic acid 8 (Stra8), one of genes induced by retinoic acid (RA), is required for the meiotic initiation of male spermatogenesis. The present study found that Stra8 inhibited apoptosis in male Stra8-knockout mice, and in mice with vitamin A deficiency and vitamin A recovery in vivo. This phenotype was also verified in $\mathrm{GC} 1$ spermatogonia (spg) cells overexpressing Stra8. In addition, microarray analysis identified that there were nine differentially expressed genes (DEGs) in the Stra8-overexpressed GC1 spg cells compared with the control groups; the expression of these nine genes was verified via mRNA expression levels. The DEGs were as follows: Phosphatidylinositol-dependent kinase 1 (PDK1), a key gene upstream of protein kinase $B$ (AKT); angiopoietin 2, a B-cell lymphoma 2 (Bcl-2)-inhibited gene; transcription factor 4, glutathione S-transferase P91 and ubiquitin-specific protease 33 , mitogen-activated protein kinase (MAPK)-related genes; oxidative stress induced growth inhibitor 1, related to the P53 pathway; Bcl-2, P53, ERK (MAPK1/3), c-Jun N-terminal kinase (MAPK8/9), and
\end{abstract}

Correspondence to: Professor Ying Zheng, Department of Histology and Embryology, School of Medicine, Yangzhou University, 11 Huaihai Road, Yangzhou, Jiangsu 225001, P.R. China E-mail: yzheng@yzu.edu.cn

${ }^{*}$ Contributed equally

Abbreviations: Stra8, stimulated by retinoic acid gene 8; RA, retinoic acid; DEGs, differentially expressed genes; VAD, vitamin A deficient; VAR, vitamin A recovery; AKT/PKB, protein kinase B; FBS, fetal bovine serum; DAPI, 4',6-diamidino-2-phenylindole; PDK1, phosphatidylinositol-dependent kinase 1; USP33, ubiquitin-specific protease 33; TCF4, transcription factor 4; ERK, extracellular signal-regulated kinase; GSTP1, glutathione S-transferase P1; ANG2, angiopoietin 2; OSGIN1, oxidative stress induced growth inhibitor 1; BIRC6, baculoviral inhibitor of apoptosis repeat-containing 6

Key words: Stra8, apoptosis, AKT, spermatogenesis
P38 (MAPK14), all of which are key genes involved in the AKT signaling pathway. Therefore, the present study further verified these genes and found that the mRNA and protein expression levels of PDK1, AKT, Bcl-2 and ERK were increased. Although the mRNA expression level of P53 was decreased, there was no significant difference in the protein expression level in Stra8-overexpressing GC1 spg cells compared with controls. In addition, Caspase 3 , one of the executioner caspases, was decreased in Stra8-overexpressing GC1 spg cells compared with the control groups. Therefore, it was suggested that Stra8 may directly or indirectly inhibit caspases through the AKT signaling pathway and ultimately exert an anti-apoptotic effect in the male reproductive system.

\section{Introduction}

Male fertility is maintained through continuous germ cell production, which are the only cells able to divide into haploid cells and carry genetic information generationally (1). Spermatozoa are produced via spermatogenesis, which is a complex process involving a series of mitosis, two stages of meiosis, and post-meiotic differentiation (2).

In mammals, meiosis is a crucial stage triggered by the metabolism of retinol and its metabolite, retinoic acid (RA), a derivative of vitamin A. RA induces the transcription and expression of stimulated by retinoic acid 8 (Stra8), which commands the initiation and progression of meiosis $(3,4)$. Stra 8 is a $45-k D a$ vertebrate-specific gene, without significant homologous proteins, expressed in the cytoplasm and nuclei of germ cells between mitosis and meiosis and presents exclusively in the embryonic and postnatal gonads of mammals. In fetal testis, RA is temporarily degraded by cytochrome P450 26B1, preventing the expression of Stra8. Following birth, RA induces the expression of Stra8 in germ cells, leading to meiotic initiation (4). In vitamin A-deficient (VAD) mice, the majority of germ cells arrest as undifferentiated spermatogonia (spg). In VAD rats, some germ cells arrest as preleptotene spermatocytes. However, when these animals are injected with RA or vitamin A, the arrested spermatogonia differentiate $(5,6)$. Stra8 gene-knockout mice are sterile, and accompanied by abnormal phenotypes, including meiotic cell cycle, chromatin condensation, homologous 
recombination, DNA repair and cell apoptosis (7-9). The testis of Stra8-defecient mice (Stra8 ${ }^{-/}$mice) contain a large number of undifferentiated spermatogonia, preleptotene spermatocytes, and spermatocytes that have features characteristic of leptonema (i.e., nuclear morphologies) (7-9). These findings indicate that Stra8 is required for transforming preleptotene spermatocytes into leptotene spermatocytes (8). According to previous reports, the phenotypes of meiotic cells are abnormal and spermatogenesis is interrupted in stra $8^{-/-}$mice, in which the majority of spermatogonia undergo apoptosis $(8,9)$; however, the mechanisms underlying the anti-apoptotic effects of Stra8 remain to be elucidated.

In the present study, it was found that Stra8 inhibited spermatogenic cell apoptosis in VAD mice, Stra $8^{-1-}$ mice and Stra8-overexpressing GC1 spg cells. Compared with the control groups, nine differentially expressed genes (DEGs) were found to be associated with apoptosis using Affymetrix GeneChip microarray analysis. Based on these results, the possible signaling pathway of Stra8 in apoptosis was discussed.

\section{Materials and methods}

Mice. The experiments included two models established in male mice: i) VAD and vitamin A recovery (VAR), respectively, on a C57BL/6 genetic background. A total of 20 VAD mice, 18 VAR mice and 36 C57BL/6J wild-type (WT) mice were used during the experiments. The mice were obtained from the Laboratory Animal Center of Yangzhou University (Yangzhou, China), and male and female mice were permitted to mate following being fed a VAD diet for 2 weeks, with the VAD diet maintained during pregnancy. Neonatal male mice were fed a VAD diet for 13-14 weeks to obtain VAD mice, and 14-week-old VAD male mice were fed a normal diet containing vitamin A to obtain VAR mice; ii) Stra8-defecicient mice on a C57BL/6 genetic background. Heterozygous B6.Cg-Stra8tm1Dcp/J mice $\left(\mathrm{Stra}^{+/ /}\right.$mice) were purchased from Jackson Laboratories (Bar Harbor, ME, USA), including one 8-week-old male mouse and two 8-week-old female mice. Stra8-deficient mice (Stra8 ${ }^{-1-}$ mice) were generated by mating heterozygotes $\left(\mathrm{Stra}^{+/-}{ }^{+-}\right.$mice). Stra8 genotypes were detected by polymerase chain reaction (PCR) as described previously (10). All mice were housed in the Laboratory Animal Center of Yangzhou University. They were housed in a controlled temperature $\left(21-25^{\circ} \mathrm{C}\right)$ and light conditions ( $12 \mathrm{~h}$ dark and $12 \mathrm{~h}$ light cycle), and fed a normal diet. The date of birth was defined as 0 days postpartum (dpp). A total of six $11 \mathrm{dpp} \mathrm{Stra8}{ }^{-/}$mice and six C57BL/6J WT mice were used for the assay and analysis of apoptosis. A total of six adult (7-8-week old) Stra ${ }^{-/-}$mice and six C57BL/6J WT mice were used for hematoxylin and eosin (H\&E) staining. The offspring were sacrificed at various postnatal ages by decapitation. All experiments were approved by the Committee on Animal Care of Yangzhou University.

$H \& E$ staining. Following sacrifice of the adult (7-8-week-old) mice by decapitation, their testes were harvested immediately and fixed with $4 \%(\mathrm{w} / \mathrm{v})$ paraformaldehyde for $24 \mathrm{~h}$, and then in chloroform overnight following dehydrating with a gradient alcohol series. The tissue was transferred into xylene and then embedded in paraffin. A microtome was used to cut the tissues into $4-\mu \mathrm{m}$ sections. Following dewaxing and hydration, the sections were stained with hematoxylin for $5 \mathrm{~min}$ and eosin for $2 \mathrm{~min}$ at room temperature. The samples were observed under a light microscope (Nikon-70i; Nikon Corporation, Tokyo, Japan).

Cell cultivation. GC1 spg cells overexpressing Stra8 (Stra8-GC1) and control GC1 spg (Control-GC1) cells were established at the laboratory at the Department of Histology and Embryology (School of Medicine, Yangzhou University) using the following procedure: i) the entire open reading frame of the Stra8 gene was inserted into lentivirus-GV34 1 (a lentiviral expression vector); ii) GV34-Stra8 was transfected into 293T cells (Shanghai Zhong Qiao Xin Zhou Biotechnology, Co., Ltd., Shanghai, China) together with two helper vectors; and iii) after 2 days, the lentiviral particles were collected and used to transfect mouse GC1 spg cells to obtain Stra8-GC1 spg cells. The lentivirus-GV34 was transfected into mouse GC1 spg cells to obtain Control-GC1 spg cells.

The Stra8-GC1 and Control-GCl cells were maintained in Dulbecco's modified Eagle's medium (Wisent, Inc., St. Bruno, QC, Canada) with $100 \mathrm{U} / \mathrm{ml}$ penicillin, $100 \mathrm{mg} / \mathrm{ml}$ streptomycin, and 10\% heat-inactivated fetal bovine serum (FBS; Hyclone, GE Healthcare Life Sciences, Logan, UT, USA), and incubated at $37^{\circ} \mathrm{C}$ with $5 \% \mathrm{CO}_{2}$. Medium exchange was performed every $48-72 \mathrm{~h}$ when the cell density reached $80-90 \%$ confluency. After $24 \mathrm{~h}$, the cells were harvested and processed for TUNEL, cell apoptosis, microarray, reverse transcription-quantitative polymerase chain reaction (RT-qPCR) and western blot analyses.

TUNEL assay. Apoptosis in the testes and germ cells was evaluated using an In Situ Cell Death Detection kit, Fluorescein (Roche Diagnostics, Basel, Switzerland) in accordance with the manufacturer's protocol. 4'6-diamidino-2-phenylindole technique (DAPI, Solarbio Science \& Technology Co., Ltd., Beijing, China) was used to visualize the level of DNA fragmentation. Following dewaxing and rehydration, the 4- $\mu \mathrm{m}$ sections of testes were incubated with a proteinase $\mathrm{K}$ working solution for $15-30 \mathrm{~min}$ at $21-37^{\circ} \mathrm{C}$. The cells were incubated in a prepared fixation solution (final concentration: 4\% PFA) for $60 \mathrm{~min}$ at $15-25^{\circ} \mathrm{C}$, and then incubated with the permeabilization solution (final concentration: $0.1 \%$ Triton X-100 with $0.1 \%$ sodium citrate) for $2 \mathrm{~min}$ on ice $\left(2-8^{\circ} \mathrm{C}\right)$, followed by two washes with PBS. The sections were incubated in $50 \mu 1$ TUNEL reaction mixture composed of label solution and enzyme solution, with label solution as a negative control. The sections were incubated in a humidified atmosphere for $60 \mathrm{~min}$ at $37^{\circ} \mathrm{C}$ in the dark. Following washing three times with PBS and then incubating with DAPI, the sections were detected and analyzed by fluorescence microscopy (Carl Zeiss AG, Oberkochen, Germany) at 515-565 nm (green light).

Cell apoptosis assay. An Annexin V/Propidium Iodide (PI) Apoptosis Detection kit (CWBIO, Beijing, China) was used to detect the apoptosis of Stra8 and control germ cells, according to the manufacturer's protocol. The cells were cultured in $60 \mathrm{~cm}$ dishes with a density of at least $1 \times 10^{6}$ cells/dish for $48 \mathrm{~h}$. The Stra8 and control germ cells were then harvested separately, and washed twice with PBS. Subsequently, $50 \mu 1$ of the cell suspension was mixed with $450 \mu 1$ Annexin $\mathrm{V}$ binding buffer, 
Table I. Primers used for microarray data validation using reverse transcription-quantitative polymerase chain reaction analysis.

\begin{tabular}{lll}
\hline Gene name & \multicolumn{1}{c}{ Forward sequence $\left(5^{\prime}-3^{\prime}\right)$} & Reverse sequence (5'-3') \\
\hline GSTP1 & CTGGAAGGAGGAGGTGGTT & TTAGATTGGTAAAGGGTGAGGT \\
BIRC6 & CGTTGGTTGTGGCAACTGAT & AAGATATAGCTGTGCCCGTAGTA \\
PDK1 & GGACTTCGGGTCAGTGAATGC & TCCTGAGAAGATTGTCGGGGA \\
USP33 & AACTGGACAAAGACAGGGAC & TGCTGATAACCGTGGACTAA \\
TCF4 & CGAAAAGTTCCTCCGGGTTTG & CGTAGCCGGGCTGATTCAT \\
OSGIN1 & CCTCCGGTATCTGCCTGTC & GGAAAGGTACTCTAGGTCCTGG \\
TEAD4 & GGCACCATTACCTCCAACG & GCTCATTCCGACCATACATCTT \\
ANG2 & GCGAAAGTATGATGGTGAAA & AGTGGTGACCTGGAAGTGAG \\
ANXA10 & CAAACAGGGAGGAAGGATA & CAAACAGGGAGGAAGGATA \\
CASP3 & CTCGCTCTGGTACGGATGTG & TCCCATAAATGACCCCTTCATCA \\
AKT1 & ATGAACGACGTAGCCATTGTG & TTGTAGCCAATAAAGGTGCCAT \\
AKT2 & ACGTGGTGAATACATCAAGACC & GGGCCTCTCCTTATACCCAAT \\
AKT3 & AGGTTGGGTTCAGAAGAGGG & AGGGGATAAGGTAAGTCCACATC \\
Bcl-2 & ATGCCTTTGTGGAACTATATGGC \\
MAPK3 (ERK2) & TCCGCCATGAGAATGTTATAGGC & GGTATGCACCCAGAGTGATGC \\
MAPK14 (P38) & TGACCCTTATGACCAGTCCTTT & GGTGGTGTTGATAAGCAGATTGG \\
TRP53 & CCCCTGTCATCTTTTGCCCT & GTCAGGCTCTTCCACTCATCTAT \\
GAPDH & GACGGCCGCATCTTCTTGT & AGCTGGCAGAATAGCTTATTGAG \\
\hline
\end{tabular}

GSTP1, glutathione S-transferase P1; BIRC6, baculoviral inhibitor of apoptosis repeat-containing 6; PDK1, phosphatidylinositol-dependent kinase 1; USP33, ubiquitin-specific protease 33; TCF4, transcription factor 4; OSGIN1, oxidative stress induced growth inhibitor 1; TEAD4, TEA domain transcription factor 4; ANG2, angiopoietin 2; ANXA10, Annexin A10; AKT, protein kinase; Bcl-2, B-cell lymphoma 2; MAPK, mitogen-activated protein kinase; CASP3, caspase 3.

and the mixture was incubated with FITC-labeled Annexin V for $5 \mathrm{~min}$, followed by PI for $10 \mathrm{~min}$ at room temperature in the dark. During the final phase of this experiment, the samples were immediately analyzed via flow cytometry (BD FACSCalibur $^{\mathrm{TM}}$, BD Biosciences, Franklin Lakes, NJ, USA).

Sample processing and microarray analysis. The microarray analysis was executed using the Affymetrix GeneChip Mouse Genome 430 2.0 Array (Affymetrix, Inc., Santa Clara, CA, USA), and 45,103 probe sets were used to analyze the expression level of at least 20,000 well-characterized mouse genes. Total RNA was isolated using the TRIzol method. The quantity and quality of RNA was determined using a NanoDrop 2000c (Thermo Fisher Scientific, Inc.) and an Agilent 2100 Bioanalyzer with an RNA 6000 Nano kit (Agilent Technologies, Inc., Santa Clara, CA, USA). Samples with an RNA integrity number $\geq 7.0$ and $260 / 280 \mathrm{~nm}$ ratios between 1.7 and 2.2 were used for detailed analyses. Total RNA was amplified, labeled and fragmented using the GeneChip 3'IVT PLUS kit and hybridized for $16 \mathrm{~h}$ at $45^{\circ} \mathrm{C}$ in a GeneChip Hybridization Oven 645 (Thermo Fisher Scientific, Inc.). The GeneChips were washed and stained in GeneChip Fluidics Station 450 and scanned with GeneChip Scanner 3000 (Affymetrix, Inc.). Differentially expressed genes (DEGs) were selected with a fold change $(\mathrm{FC})>1.5$ and $\mathrm{P}<0.01$ (one-sample t-test). A functional analysis based on the Kyoto Encyclopedia of Genes and Genomes (KEGG) pathway database (https://www.kegg. $\mathrm{jp} /$ ) and Gene Ontology (http://www.geneontology.org/) was accomplished.
Reverse transcription-quantitative polymerase chain reaction $(R T-q P C R)$ assay. For RT-qPCR analysis, total RNA was isolated from the Stra8 germ cells and control germ cells using the traditional TRIzol method and reverse transcribed with the PrimeScript ${ }^{\mathrm{TM}}$ RT reagent kit with gDNA Eraser (Takara Bio, Inc., Otsu, Japan). The forward and reverse PCR primers were designed by Wcgene Biotech Company (Shanghai, China) in accordance with the PCR conditions (listed in Table I). The $10 \mu \mathrm{l}$ RT-qPCR reaction mix contained $5 \mu \mathrm{l}$ 2X RNA GoTaq ${ }^{\circledR} \mathrm{qPCR}$ Master mix (Promega Corporation, Madison, WI, USA), $10 \mathrm{pmol}$ of the forward and the reverse primers, and $0.5 \mu \mathrm{l}$ of the sample/standard. The PCR thermal profile included 40 cycles of denaturation at $95^{\circ} \mathrm{C}$ for $15 \mathrm{sec}$, annealing at $60^{\circ} \mathrm{C}$ for $30 \mathrm{sec}$, and extension at $72^{\circ} \mathrm{C}$ for $30 \mathrm{sec}$. Each sample was analyzed in triplicate. The mRNA level of GAPDH was used as the internal control, and relative gene expression was calculated according to the $2^{-\Delta \Delta \mathrm{Cq}}$ method based on the quantification cycle values (11).

Western blot analysis. Whole proteins from the Stra8-GC1 and Control-GC1 cells were prepared in RIPA buffer (Solarbio Science \&Technology Co., Ltd.) and quantified by using the BCA Protein Assay kit (CWBIO). The homogenates were centrifuged for $15 \mathrm{~min}$ at $10,000 \mathrm{xg}$ at $4^{\circ} \mathrm{C}$ and the supernatants were collected. Equal quantities of protein $(60 \mu \mathrm{g} /$ lane $)$ were separated by $10 \%$ sodium dodecyl sulfatepolyacrylamide gel electrophoresis and transferred onto a polyvinylidene difluoride membrane. The membrane was blocked with Tris-buffered saline (TBS) with 5\% non-fat milk and incubated with the 
A
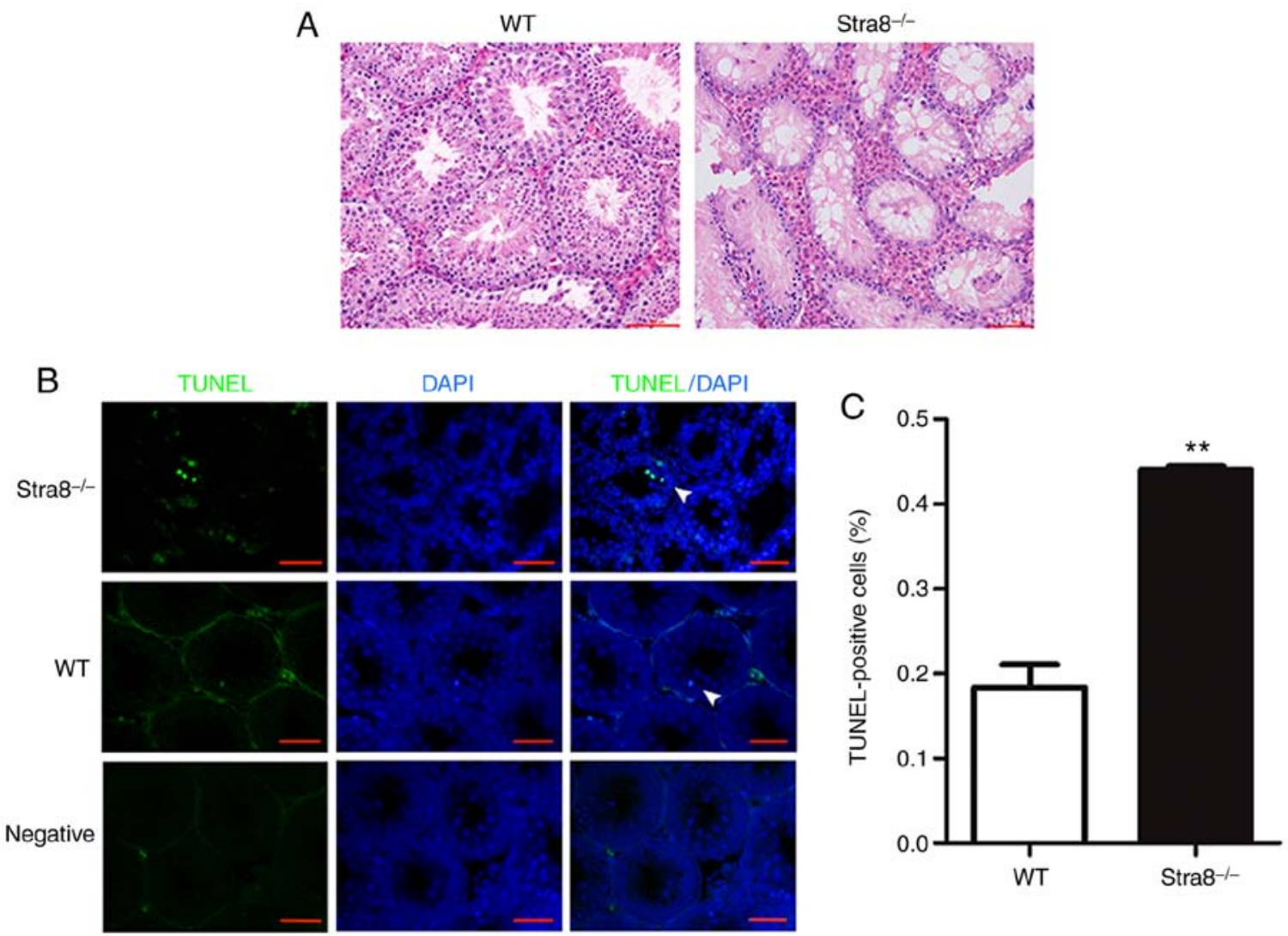

Figure 1. Anti-apoptotic effect of Stra8 in male Stra8 ${ }^{-/}$and WT mice. (A) Adult WT and Stra8-defecient mice (Stra8 $8^{-1-}$ ) testes stained with hematoxylin and eosin. Scale bar $=100 \mu \mathrm{m}$. (B) Top and middle rows show the fluorescence images of TUNEL/DAPI from the seminiferous tubules in the testes of Stra8 $8^{-/}$and WT mice, respectively; bottom rows show the negative fluorescence images. White arrows indicate TUNEL-positive cells. Fluorescence colors show TUNEL in green and DAPI in blue; scale bar=200 $\mu \mathrm{m}$. (C) Percentage of apoptotic cells (TUNEL-positive cells/DAPI-positive cells) among the seminiferous tubules. $\mathrm{N} \geq 6$. Error bars show the mean \pm standard deviation. ${ }^{* *} \mathrm{P}<0.01$ (two-tailed Student's t-test). Stra8, stimulated by retinoic acid gene 8; WT, wild-type; DAPI, 4',6-diamidino-2-phenylindole.

primary detection antibodies: Phosphatidylinositol-dependent kinase 1 (PDK1; cat. no. sc-293160, Santa Cruz Biotechnology, Inc., CA, USA; 1:1,000), Phospho-AKT (cat. no. bs-0876R; BIOSS, Beijing, China, 1:500), B-cell lymphoma 2 (Bcl-2; cat. no. 3498T; Cell Signaling Technology, Inc., Beverly, MA, USA, 1:1,000), Mitogen-activated protein kinase (MAPK) Family Antibody Sampler kit for extracellular signal-regulated kinase (ERK)1/2, c-Jun N-terminal kinase (JNK)1/2, and P38 (cat. no. 9926T; Cell Signaling Technology, Inc., Beverly, MA, USA, 1:1,000), P53 (cat. no. sc-6243, Santa Cruz Biotechnology, Inc.; 1:1,000), cleaved caspase 3 (cleaved CASP3; cat. no. 9664T, Cell Signaling Technology, Inc.; 1:1,000), GAPDH (cat. no. CW0100, CWBIO; $1: 5,000$ ) at $4^{\circ} \mathrm{C}$ overnight. Following rinsing three times with TBS/Tween-20, the membranes were incubated with the following secondary antibodies: Peroxidase-Conjugated Goat anti-Rabbit IgG (cat no. ZB-2301; OriGene Technologies, Inc., Rockville, MD, USA; 1:1,000) and Peroxidase-Conjugated Goat anti-Mouse IgG (cat no. ZB-2305; OriGene Technologies, Inc.; 1:1,000) at room temperature for $1.5 \mathrm{~h}$. Protein levels were normalized to GAPDH level and all values were presented compared with the $\mathrm{NC}$, which was normalized as 1 . All proteins were detected with an electrogenerated chemiluminescence assay (Tanon-5200; Tanon Science and Technology Co., Ltd. Shanghai, China) and the Gel Image System, version 4.00 (Tanon Science and Technology Co., Ltd.).
Statistical analysis. Statistical analysis was performed using Microsoft Excel 2010 (Microsoft Corporation, Redmond, WA USA). All data are expressed as the mean \pm standard deviation of at least three independent experiments. To compare between two groups, an unpaired Student's t-test (one- or two-tailed as indicated) was used. $\mathrm{P}<0.05$ was considered to indicate a statistically significant difference.

\section{Results}

Stra8 exhibits an anti-apoptotic effect in Stra $8^{-/}$male mice. $\mathrm{Stra}^{-/ /}$mice were generated through mating heterozygotes, and tissue sections were stained with H\&E (Fig. 1A). Compared with the WT mice, the number of spermatogonia in the seminiferous tubules of the Stra $8^{-/}$mice began decreasing from

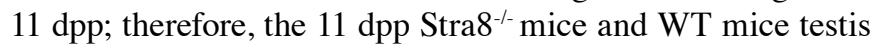
were selected for the TUNEL assay to evaluate the number of apoptotic germ cells in the seminiferous tubules, separately. The results from the TUNEL assay suggested that the number of apoptotic germ cells in the Stra8 $8^{-1-}$ mice was significantly increased compared with that in the WT mice (Fig. 1B). The apoptotic index was $0.18 \pm 0.06$ in the WT mice, compared with $0.44 \pm 0.01$ in the Stra8 ${ }^{-/}$mice (Fig. 1C).

Anti-apoptotic effect of Stra8 in VAD and VAR mice. In addition, the anti-apoptotic effect of Stra8 was verified in a 
A
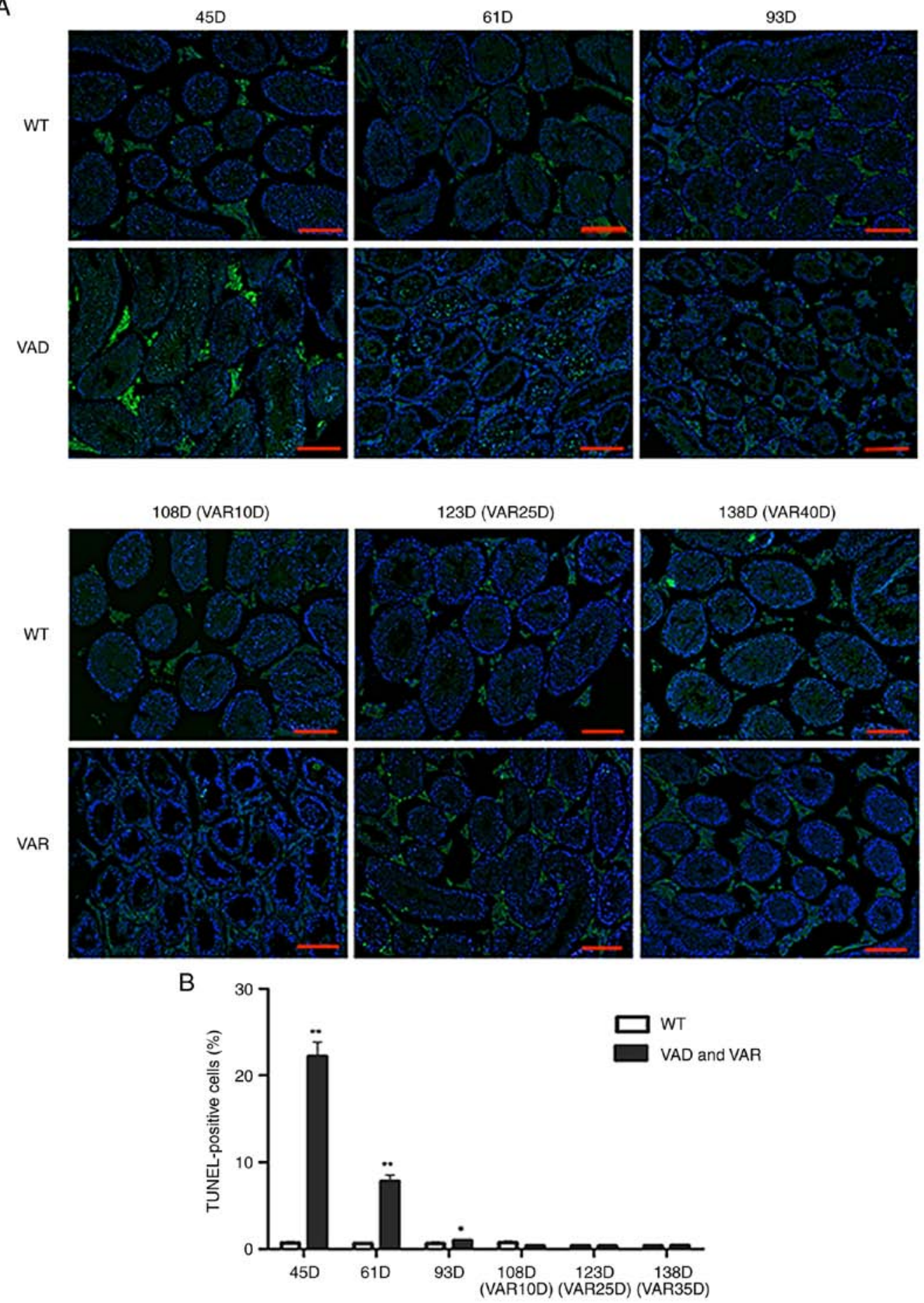

Figure 2. Anti-apoptotic effect of Stra8 in male VAD and VAR mice. (A) Representative images of TUNEL/DAPI staining from the seminiferous tubules in the testes of male WT mice, and VAD and VAR mice at 45, 61, 93, 108 (VAR 10), 123 (VAR 25), and 138 (VAR 40) days. Fluorescence colors show TUNEL in green and DAPI in blue; scale bar=100 $\mu \mathrm{m}$. (B) Percentage of apoptotic cells (TUNEL-positive cells/DAPI-positive cells) among the seminiferous tubules. $\mathrm{N} \geq 6$. The data are presented as the mean \pm standard deviation. ${ }^{*} \mathrm{P}<0.05,{ }^{* *} \mathrm{P}<0.01$ (two-tailed Student's t-test). Stra8, stimulated by retinoic acid gene 8 ; WT, wild-type; DAPI, 4',6-diamidino-2-phenylindole; VAD, vitamin A deficient; VAR, vitamin A recovery.

male VAD and VAR mouse model. Male and female mice were permitted to mate following being fed a VAD diet for 2 weeks, with the VAD diet maintained during pregnancy. Neonatal male mice were fed a VAD diet for 13-14 weeks to obtain VAD mice. According to morphological observations, there was no phenotypic difference at 45 days in the VAD mice compared with the WT mice; however, the number of germ cells was significantly decreased in the testis of mice at
61 days; and only Sertoli cells, a small number of germ cells, and a large number of vacuoles in the testes exhibiting structural disorganization were observed at 93 days. Therefore, 45 , 61, and 93 days were selected as the time points to evaluate VAD and WT mice using a TUNEL assay. The results showed that the numbers of TUNEL-positive cells in the VAD mice on days 45 and 61 were higher than those in the WT mice (Fig. 2A). The numbers of TUNEL-positive cells were as 
Table II. Average number of TUNEL-positive cells per tubule from the WT, VAD and VAR mice.

\begin{tabular}{lrccccc}
\hline Group & $45 \mathrm{D}$ & $61 \mathrm{D}$ & $93 \mathrm{D}$ & $108 \mathrm{D}$ (VAR 10D) & 123 D (VAR 25D) & $138 \mathrm{D}$ (VAR 40D) \\
\hline Wild-type & $0.698 \pm 0.010$ & $0.626 \pm 0.030$ & $0.636 \pm 0.050$ & $0.729 \pm 0.050$ & $0.401 \pm 0.040$ & $0.381 \pm 0.030$ \\
Model & $22.224 \pm 2.304$ & $7.815 \pm 0.999$ & $0.979 \pm 0.090$ & $0.395 \pm 0.050$ & $0.354 \pm 0.030$ & $0.415 \pm 0.048$ \\
\hline
\end{tabular}

Data are presented as the mean \pm standard deviation $(n=120)$. D, day; WT, wild-type; VAD, vitamin A-deficient; VAR, vitamin A recovery.
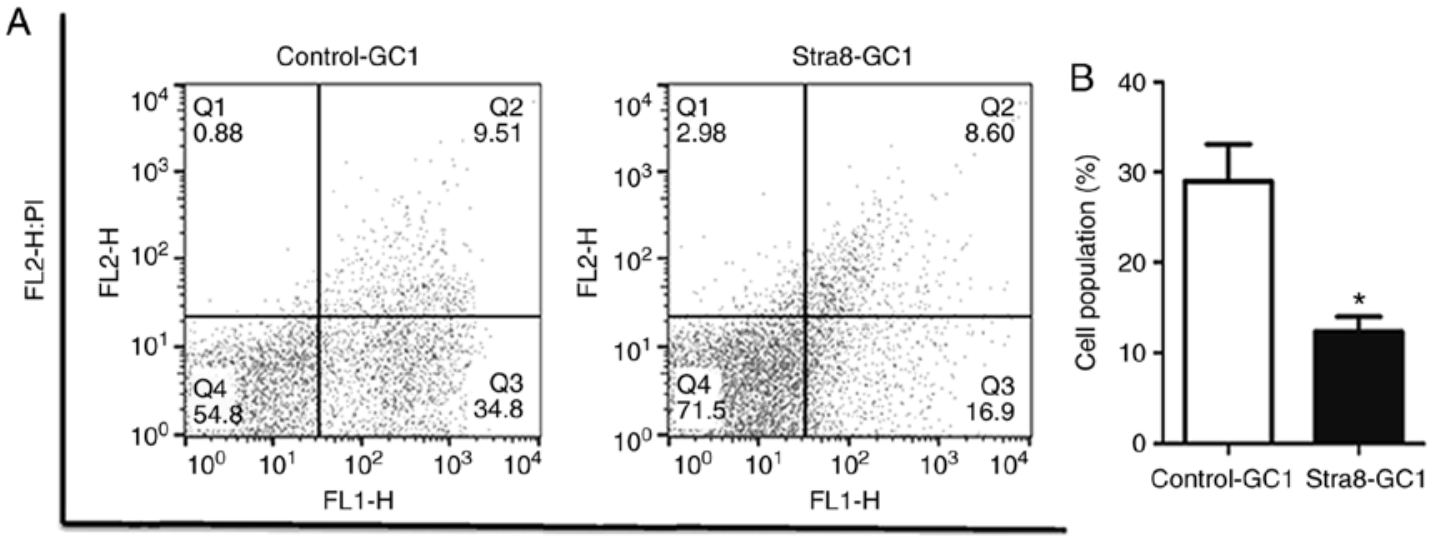

FL1-H: Annexin V
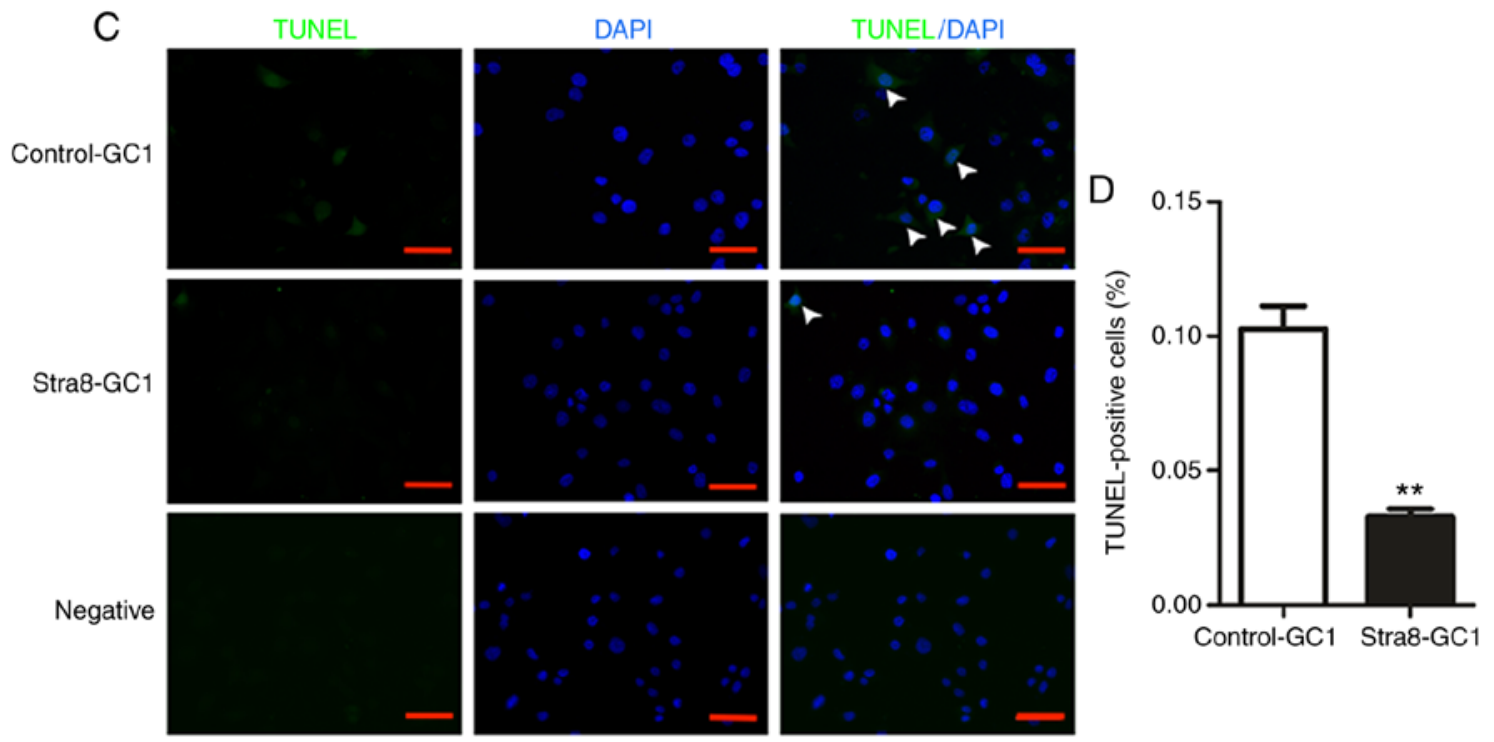

Figure 3. Anti-apoptotic effect of the overexpression of Stra8 on GC1 spg. (A) Apoptotic cells in the Stra8-GC1 and Control-GC1 groups were measured by flow cytometry and the cells were divided into four sections: Q1, mechanical error; Q2, late apoptosis cells; Q3, early apoptotic cells; and Q4, living cells. (B) Percentage of cells in early apoptosis in the two groups $(\mathrm{N} \geq 6$ ). Error bars show the mean \pm standard deviation. * $\mathrm{P}<0.05$ (two-tailed Student's $\mathrm{t}$-test). (C) Top and middle rows show the fluorescence images of TUNEL/DAPI in Control-GC1 and Stra8-GC1 cells, respectively; bottom row shows the negative fluorescence images. White arrows indicate TUNEL-positive cells. Fluorescence colors show TUNEL in green and DAPI in blue; scale bar=200 $\mu \mathrm{m}$. (D) Percentage of apoptotic cells (TUNEL-positive cells/DAPI-positive cells) among the total cells. $n=3$ /group. Error bars indicate the mean \pm standard deviation. ${ }^{* *} \mathrm{P}<0.01$ (two-tailed Student's t-test). ). Stra8, stimulated by retinoic acid gene 8; WT, wild-type; DAPI, 4',6-diamidino-2-phenylindole; spg, spermatogonia; PI, propidium iodide.

follows: $0.69 \pm 0.01$ in WT mice, $22.22 \pm 2.304$ in VAD mice (45 days); $0.62 \pm 0.03$ in WT mice, $7.81 \pm 0.99$ in VAD mice (61 days); $0.63 \pm 0.05$ in WT mice; and $0.97 \pm 0.09$ in VAD mice (93 days) (Fig. 2B; Table II).

Subsequently, the 14-week-old VAD male mice were fed a normal vitamin A diet to obtain VAR mice. According to the morphological observations, spermatocytes appeared in seminiferous tubules in the testis of VAR mice following 10 days on the normal diets, different types of spermatogenic cells in the seminiferous tubules had recovered at 25 days, and the seminiferous tubules exhibited a normal structure at 40 days. Therefore, at 10, 25, and 40 days, VAR mice were selected for further verification using a TUNEL assay; however, no differences were observed between the VAR and WT mice (Fig. 2A). The respective number of TUNEL-positive cells in the WT and VAR mice were as follows: $0.72 \pm 0.05$, vs. $0.39 \pm 0.05$ (10 days); $0.40 \pm 0.04$, vs. $0.35 \pm 0.03$ (25 days); and $0.38 \pm 0.03$, vs. $0.41 \pm 0.04$ (40 days) group (Fig. 2B; Table II). 


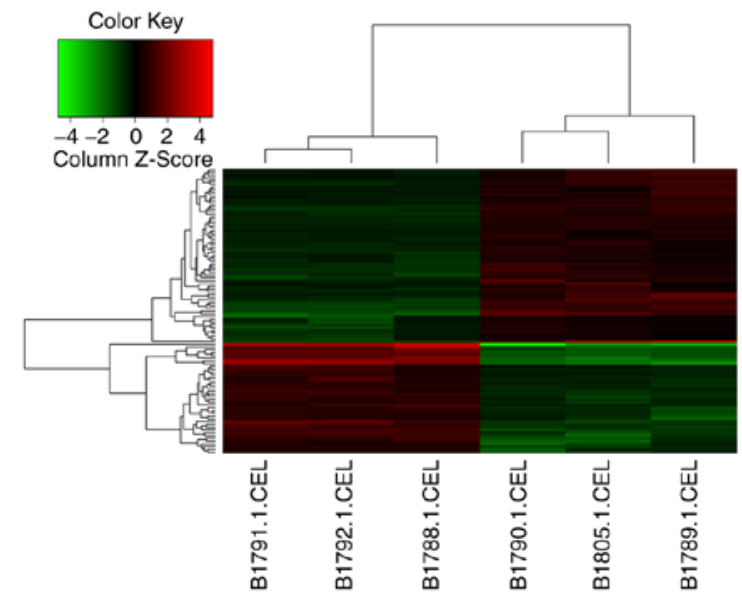

Figure 4. GeneChip microarray analysis of the overexpression of Stra8 on spg- and control-GC1 cells. The heat maps of the differentially expressed genes in the Stra8-GC1 and Control-GC1 cells. The red color indicates upregulated genes and the green color indicates downregulated genes. Stra8, stimulated by retinoic acid gene 8; spg, spermatogonia.

Overexpression of Stra8 inhibits cellular apoptosis of GCI spg cells. To further assess the anti-apoptotic effect of Stra8 in vitro, analyses of Stra8-GC1 and Control-GC1 were performed using an Annexin V/PI apoptosis detection kit via flow cytometry. These results demonstrated that the proportion of Stra8-GC1 cells that underwent early apoptosis was lower than that in the Control-GC1 cells (Fig. 3A). Apoptotic germ cells comprised $29.52 \pm 10.53$ of the population in the Control-GC1 cells and $12.35 \pm 4.41$ in the Stra8-GC1 cells (Fig. 3B).

Additionally, the results of the TUNEL assay confirmed the anti-apoptotic effect of Stra8 in germ cells. The number of apoptotic germ cells was significantly decreased when Stra8 was overexpressed compared with the control group (Fig. 3C). The apoptotic index was $0.10 \pm 0.01$ in the Control-GC1 cells compared with $0.03 \pm 0.04$ in the Stra8-overexpressing GC1 cells (Fig. 3D).

Anti-apoptotic effect of Stra8 in GC1 spg cells overexpressing Stra8 according to Affymetrix GeneChip microarray analysis. To investigate which genes or pathways were involved in the anti-apoptotic effect of Stra8, an Affymetrix GeneChip microarray of the Stra8-GC1 and Control-GC1 cells was completed. A total of 45,103 probe sets were used to analyze the expression level of $>20,000$ well-characterized mouse genes using the Affymetrix Mouse Genome 430 2.0 Array. DEGs were selected with FC $>1.5$ and $\mathrm{P}<0.01$ (one-sample t-test). A heat map illustrates the differential expression patterns between the clustering of the genes in the Stra8-GC1 cells compared with that of the Control-GC1 cells (Fig. 4).

Based on the analysis of DEGs using the KEGG pathway database and Gene Ontology, the biological processes involved in DEGs included the negative regulation of apoptosis, negative regulation of programmed cell death, system development, structure development, and the negative regulation of cellular processes. The top 20 upregulated and downregulated genes were selected and displayed according to the P-values (Table III). Among all the DEGs, five upregulated genes [glutathione S-transferase P91 (GSTP1), baculoviral inhibitor of apoptosis repeat-containing 6 (BIRC6), PDK1, ubiquitin-specific protease 33 (USP33), and transcription factor 4 (TCF4)]; and four downregulated genes [(oxidative stress induced growth inhibitor 1 (OSGIN1), TEA domain transcription factor 4 (TEAD4), angiopoietin 2 (ANG2), and Annexin A10 (ANXA10)] associated with apoptosis were selected according to evidence obtained from references relating to gene function (Table IV).

Potential signaling pathways involved in the anti-apoptotic effect mediated by Stra8. RT-qPCR analysis was performed to verify the tendency of the nine genes described above, the results of which were consistent with the GeneChip microarray analysis. The genes in the caspase family were also examined and it was found that CASP3 was reduced in Stra8-GC1 cells (Fig. 5A). Through a literature review, it was found that PDK1, TCF4, GSTP1, USP33, ANG2, and OSGIN1 are associated with the AKT signaling pathway. Among these genes, PDK1 is a key upstream factor of AKT; therefore, the present study verified the mRNA and protein expression levels of PDK1 and AKT using RT-qPCR and a western blot analyses. It was found that the levels of PDK1 and AKT were increased in Stra8-GC1 cells compared with those in Control-GC1 cells, which is consistent with the hypothesis that Stra8 may exert an anti-apoptotic effect through the AKT pathway (Fig. 5B-D).

ANG2 is a Bcl-2-inhibited gene; TCF4, GSTP1 and USP33 are MAPK-related genes; and OSGIN1 is related to the P53 pathway. In addition, Bcl-2, p53, ERK (MAPK1/3), JNK (MAPK8/9), and P38 (MAPK14) are key genes involved in the AKT signaling pathway. Therefore, the mRNA expression levels of these genes were determined, which revealed that Bcl-2 and ERK were increased, whereas P53 was decreased, in the Stra8-GC1 cells compared with the Control-GC1 cells. In addition, the protein expression levels of Bcl-2 and ERK were increased, and there was no significant difference in the expression of P53 in the Stra8-GC1 cells compared with the control groups. In addition, CASP3, one of the executioner caspases, was decreased in the Stra8-GC1 cells compared with the Control-GC1 at the mRNA and protein expression levels (Fig. 5-D). Therefore, it was concluded that Stra8 may exert an anti-apoptotic effect primarily through the AKT pathway (Fig. 6).

\section{Discussion}

Previous publications have described Stra8 as an RA-inducible gene, which is specifically expressed during mouse embryogenesis, is involved in the premeiotic phase of spermatogenesis, and is also required for early meiotic events and the transformation from mitosis to meiosis $(7,12)$. In previous experiments, whether Stra8 can affect spermatogenic cell proliferation was examined using an SRB cell proliferation assay. The cells were collected and counted at $4 \mathrm{~h}$ (day 0 ), and on days 1, 2, 3, 4 , and 5, and a growth curve was plotted using the optical density (OD) value every $24 \mathrm{~h}$. No statistical difference in OD values was found at corresponding time points between the Stra8-GC1 and Control-GC1 (data not shown). Furthermore, using flow cytometry, the cell numbers of different stages of the cell cycle were detected, which revealed no statistically 
Table III. Top 20 upregulated and downregulated genes of the total differentially expressed genes according to the P-values.

\begin{tabular}{|c|c|c|c|c|}
\hline No & Gene symbol & Description & Fold change & $\mathrm{P}$-value \\
\hline \multicolumn{5}{|c|}{ Upregulated } \\
\hline 1 & BGN & Biglycan & 3.5835900 & 0.012659261 \\
\hline 2 & ANK2 & Ankyrin 2 & 3.2059138 & 6.80039E-06 \\
\hline 3 & GJB5 & Gap junction protein, $\beta 5$ & 2.4792826 & $6.03425 \mathrm{E}-06$ \\
\hline 4 & AI256396 & Est ai256396 & 2.3620548 & 0.000472130 \\
\hline 5 & GSTP1 & Glutathione S-transferase pi 1 & 2.2966921 & 0.003767367 \\
\hline 6 & RBM12B1 & RNA binding motif protein $12 \mathrm{~B} 1$ & 2.2234132 & 0.000175803 \\
\hline 7 & CTH & Cystathionase & 2.2095652 & 0.003464704 \\
\hline 8 & OPLAH & 5-oxoprolinase (ATP-hydrolysing) & 2.0994134 & 0.016354447 \\
\hline 9 & MBP & Myelin basic protein & 1.9661545 & 0.001004058 \\
\hline 10 & VAT1L & Vesicle amine transport protein 1 like & 1.9652303 & 0.033937313 \\
\hline 11 & SLC38A9 & Solute carrier family 38, member 9 & 1.9384620 & 0.001569600 \\
\hline 12 & ZFP422 & Zinc finger protein 422 & 1.9111286 & 0.000111407 \\
\hline 13 & KLF9 & Kruppel-like factor 9 & 1.8877469 & 0.005244654 \\
\hline 14 & MANBA & Mannosidase, $\beta \mathrm{A}$, lysosomal & 1.8711381 & 0.000459721 \\
\hline 15 & CBLN1 & Cerebellin 1 precursor protein & 1.8617241 & 0.000945486 \\
\hline 16 & TRPM3 & $\begin{array}{l}\text { Transient receptor potential cation channel, } \\
\text { subfamily } \mathrm{M} \text {, member } 3\end{array}$ & 1.8470881 & 0.015302277 \\
\hline 17 & GNPDA2 & Glucosamine-6-phosphate deaminase 2 & 1.8342222 & 0.004544168 \\
\hline 18 & ZFP467 & Zinc finger protein 467 & 1.8330021 & 0.028772602 \\
\hline 19 & RNF113A2 & Ring finger protein $113 \mathrm{~A} 2$ & 1.8240016 & $2.24117 \mathrm{E}-07$ \\
\hline 20 & DMRT2 & $\begin{array}{l}\text { Doublesex and mab-3 related transcription } \\
\text { factor } 2\end{array}$ & 1.8220981 & 0.000109843 \\
\hline
\end{tabular}

Downregulated

\begin{tabular}{|c|c|}
\hline 1 & KRT6A \\
\hline 2 & ANG2 \\
\hline 3 & SERPINI2 \\
\hline 4 & CD302 \\
\hline כ & IGFBP7 \\
\hline 6 & EYA2 \\
\hline & SMAGP \\
\hline & TMEM181C-PS \\
\hline & TMEM181A \\
\hline & TMEM181B-PS \\
\hline & OSGIN1 \\
\hline & SLC7A2 \\
\hline & ASS1 \\
\hline & CTSH \\
\hline & CRYAB \\
\hline & FLT1 \\
\hline & PTPRG \\
\hline & ANXA10 \\
\hline & RANGRF \\
\hline 0 & APCDD1 \\
\hline
\end{tabular}

Keratin 6A

Angiogenin,ribonuclease A family,member 2

Serine (or cysteine) peptidase inhibitor,

clade I, member 2

CD302 antigen

Insulin-like growth factor binding protein 7

EYA transcriptional coactivator and

phosphatase 2

Small cell adhesion glycoprotein

Tmem181c-ps transmembrane protein

181C, pseudogene

Transmembrane protein 181A

Tmem181b-ps transmembrane protein

181B, pseudogene

Oxidative stress induced growth inhibitor 1

Solute carrier family 7

Argininosuccinate synthetase 1

Cathepsin $\mathrm{H}$

Crystallin, $\alpha \mathrm{B}$

FMS-like tyrosine kinase 1

Protein tyrosine phosphatase, receptor type, G

Annexin A10

RAN guanine nucleotide release factor

Adenomatosis polyposis coli downregulated 1

$\begin{array}{ll}-10.943147 & 0.000540374 \\ -5.6302330 & 0.000515156 \\ -5.3056600 & 8.79068 \mathrm{E}-05 \\ -4.6146510 & 0.000681903 \\ -4.0378250 & 8.85925 \mathrm{E}-05 \\ -3.1538005 & 1.15442 \mathrm{E}-06 \\ -2.9982140 & 2.30374 \mathrm{E}-06 \\ -2.9360726 & 0.000162202 \\ -2.9360726 & 0.000162202 \\ -2.9360726 & 0.000162202 \\ -2.5084667 & 0.001495294 \\ -2.3679860 & 0.002371071 \\ -2.2645633 & 0.000348439 \\ -2.2382452 & 0.000414636 \\ -2.1004624 & 0.002057886 \\ -2.0917470 & 0.009599945 \\ -2.0450683 & 0.000267192 \\ -2.0104885 & 0.000560119 \\ -1.8825512 & 0.015963247 \\ -1.8573459 & 6.23783 \mathrm{E}-05 \\ & \\ & \\ & \\ & \\ & \\ & \\ & \\ & \end{array}$


Table IV. Selected upregulated and downregulated genes among all differentially expressed genes.

\begin{tabular}{lllll}
\hline No & Gene symbol & \multicolumn{1}{c}{ Description } & Fold change & P-value \\
\hline Upregulated & & & & \\
1 & GSTP1 & Glutathione S-transferase pi 1 & 2.2966921 & 0.003767367 \\
2 & BIRC6 & Baculoviral IAP repeat-containing 6 & 1.7460216 & 0.000302024 \\
3 & PDK1 & Pyruvate dehydrogenase kinase 1 & 1.7768697 & 0.006251959 \\
4 & USP33 & Ubiquitin specific peptidase 33 & 1.5531131 & 0.000201205 \\
5 & TCF4 & Transcription factor 4 & 1.6565648 & 0.000173130 \\
Downregulated & & & & \\
1 & OSGIN1 & Oxidative stress induced growth inhibitor 1 & -2.5084667 & 0.001495294 \\
2 & EPHA4 & Eph receptor A4 & -1.5859284 & 0.007054214 \\
3 & ANG2 & Angiogenin, ribonuclease A family, member 2 & -5.630233 & 0.000515156 \\
4 & ANXA10 & Annexin A10 & -2.0104885 & 0.000560119 \\
\hline
\end{tabular}

significant difference between the Stra8-GC1 and Control-GC1 groups (data not shown). Therefore, it was hypothesized that Stra8 may have no influence on the proliferation or cell cycle of spermatogenic cells. In the present study, using a TUNEL assay, a routine and normal method for visualizing cells undergoing apoptosis, it was found that Stra8 inhibited spermatogenic cell apoptosis. Furthermore, this anti-apoptotic effect of Stra8 was verified using male VAD and VAR mice models. On days 45 and 61, the number of apoptotic cells in the VAD mice was significantly increased compared with that in the WT mice on the same days, which was consistent with expectations. However, no differences were observed at 93 days between the VAD and WT mice. This may be due to the reduction in the total number of spermatogenic cells in the seminiferous tubules at 93 days in the VAD mice, in which severe vacuolization and disorganized seminiferous tubules were observed. In addition, a moderate expression of Stra8 remained in the seminiferous tubules within 10 days in the testis of the VAR mice, which was sufficient to mediate an anti-apoptotic effect. The expression level of Stra8 had returned to normal within 40 days in the VAR mice testis, therefore, no significant difference in the number of apoptotic cells was observed between the WT and VAR mice. The present study further verified the anti-apoptotic effect of Stra8 in vitro using Stra8-overexpressing GC1 spg cells. To determine the potential genes and possible pathways of the Stra8 anti-apoptotic effects, which have not been previously reported, GeneChip microarray analysis was performed. A total of 75 upregulated genes and 46 downregulated genes were found through the GeneChip microarray of the Stra8-GC1 and Control-GC1 cells. Among these DEGs, five upregulated genes and four downregulated genes were associated with apoptosis. Additional analyses on the function and related pathways of these nine genes were performed to obtain further understanding of the anti-apoptotic effect of Stra8.

Apoptosis is the process of programmed cell death, which is critical in spermatogenesis and the elimination of germ cells that carry DNA ns mutations (13). A large number of studies have indicated that several pathways are involved in apoptosis during germ development, including the Fas/Fas ligand system (13), MAPK signal pathways, Bcl-2 gene fami- lies $(14,15)$, P53, heat shock protein 70 (16) and AKT signaling pathways (17). Fas/Fasl are expressed in Sertoli and spermatogenetic cells, and increase under estrogen exposure, inducing cell $\mathrm{h}$ death (13). In addition, Bcl-2 can prevent germ cell death during spermatogenesis $(14,15)$. Furthermore, under genotoxic stress, p53, a sequence specific transcription factor, is involved in the apoptosis of spermatogonia, spermatocytes and spermatids, and can regulate the expression of Bcl-2 (16). Abraham et al (18) demonstrated that AKT negatively regulates levels of p53 by enhancing murine double minute 2 . In addition, previous data show that p53 is involved in promoting apoptosis by stimulating the release of pro-apoptotic proteins, including cytochrome $c$, second mitochondria-derived activator of caspase, and apoptosis-inducing factor, and the activation of caspases (Casp3, 6, and 7) (19,20). In addition, MAPK signaling has a key functional role in numerous male reproductive processes, including spermatogenesis, capacitation and the acrosome reaction prior to $n$ fertilization (21). AKT signaling has an anti-apoptotic effect as a survival factor, and the inhibition of AKT results in DNA replication damage and cell cycle arrest, leading to $\mathrm{s}$ apoptosis $(22,23)$. Furthermore, phosphoinositide 3-kinase (PI3K)-AKT signaling leads to cellular proliferation, survival and growth (23). According to previous studies, RA activates the PI3K-AKT signaling network to regulate the differentiation of spermatogonia in neonatal testes (24). In addition, Busada et al (25) found that mTOR complex 1 is required to mediate the RA signal to direct spermatogonial differentiation. Therefore, PI3K-AKT signaling is essential for male reproduction. Stra8 is one of the genes induced by RA that shuttles between the cytoplasm and nucleus, depending on the different dynamics of each cell type, and may exert distinct functions in various cellular compartments (1). Therefore, the function of Stra 8 may be associated with different cell cycles. Additionally, the majority of DEGs associated with apoptosis exhibiting an overexpression of Stra8 GC1 spg are related to AKT signaling, including PDK1, USP33, TCF4, GSTP1, ANG2, OSGIN1 and CASP3. Therefore, it was hypothesized that Stra8 may have an apoptotic role associated with the AKT pathway.

Based on the above results, the present study further examined the function of AKT-associated genes and pathways. It has 

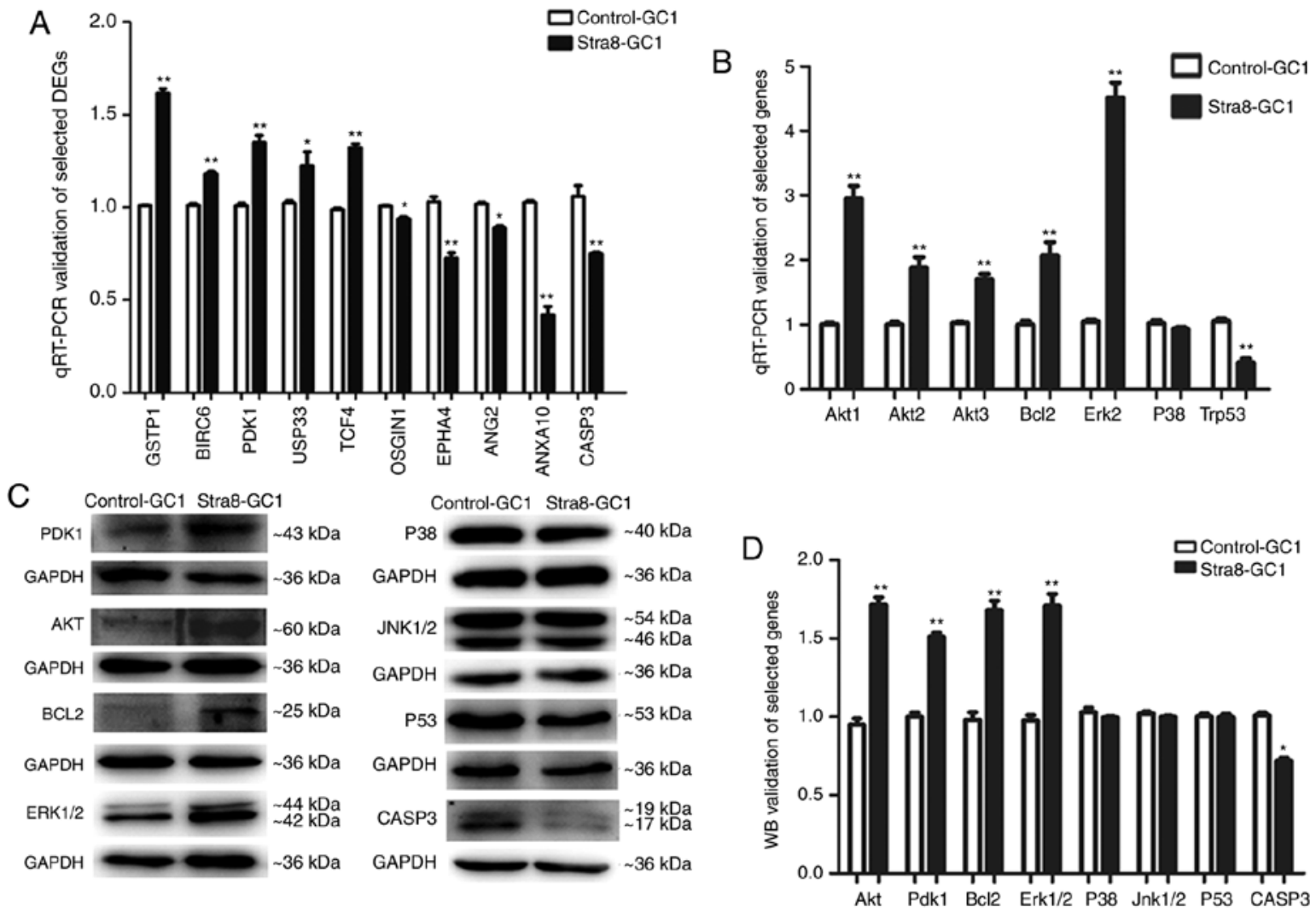

Figure 5. Potential signaling pathways by which Stra8 mediates an anti-apoptotic effect. (A) Nine DEGs were associated with apoptosis in Stra8-GC1 cells compared with Control-GC1 cells, determined through Affymetrix GeneChip microarray analysis. Among these DEGs, five upregulated genes (GSTP1, BIRC6, PDK1, USP33, and TCF4) and four downregulated genes (OSGIN1, TEAD4, ANG2, and ANXA10) were identified through RT-qPCR analysis. CASP3, one of known executioner caspases, was downregulated in Stra8-GC1 cells. N=3/group. Error bars show the mean \pm standard deviation, ${ }^{*} \mathrm{P}<0.05$, ${ }^{* *} \mathrm{P}<0.01$ (two-tailed Student's t-test). (B) RT-qPCR analysis of key genes (AKT1, AKT2, AKT3, Bcl-2, ERK2, P38 and Trp53) of the AKT pathway in Stra8-GC1 cells compared with Control-GC1 cells. (C) Western blot of key genes (PDK1, AKT, Bcl-2, ERK1/2, P38, JNK1/2, P53, CASP3) of the AKT pathway in Stra8-GC1 cells compared with Control-GC1 cells. (D) Western blot grayscale value analysis of key genes (PDK1, AKT, Bcl-2, ERK1/2, P38, JNK1/2, P53, CASP3) of the AKT pathway; $\mathrm{N} \geq 3$. Error bars show the mean \pm standard deviation ${ }^{*} \mathrm{P}<0.05,{ }^{* *} \mathrm{P}<0.01$ (two-tailed Student's $\mathrm{t}-$ test). Stra8, stimulated by retinoic acid gene 8; DEGs, differentially expressed genes; RT-qPCR, reverse transcription-quantitative polymerase chain reaction; WB, western blotting; GSTP1, glutathione S-transferase P1; BIRC6, baculoviral inhibitor of apoptosis repeat-containing 6; PDK1, phosphatidylinositol-dependent kinase 1; USP33, ubiquitin-specific protease 33; TCF4, transcription factor 4; OSGIN1, oxidative stress induced growth inhibitor 1; TEAD4, TEA domain transcription factor 4; ANG2, angiopoietin 2; ANXA10, Annexin A10; AKT, protein kinase; Bcl-2, B-cell lymphoma 2; ERK, extracellular signal-regulated kinase; JNK, c-Jun N-terminal kinase; CASP3, caspase 3.

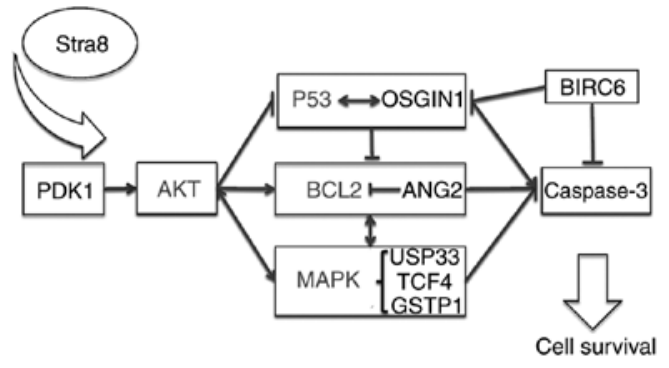

Figure 6. Possible pathway through which Stra8 may exert an anti-apoptotic effect via the AKT signaling pathway. Stra8, stimulated by retinoic acid gene 8; PDK1, phosphatidylinositol-dependent kinase 1; AKT, AKT, protein kinase; OSGIN1, oxidative stress induced growth inhibitor 1; Bcl-2, B-cell lymphoma 2; ANG2, angiopoietin 2; MAPK, mitogen-activated protein kinase; USP33, ubiquitin-specific protease 33; TCF4, transcription factor 4; GSTP1, glutathione S-transferase P1; BIRC6, baculoviral inhibitor of apoptosis repeat-containing 6 .

been shown that AKT acts as a survival factor by phosphorylating pro-apoptotic substrates, including Bcl-2-associated death promotor, CASP9, forkhead box O, and apoptosis signal-regu- lating kinase $1(22,26,27)$. According to associated studies in the literature, AKT can act as a survival factor which balances pro-survival and pro-death signals, including activating genes in the Bcl-2 family and interacting with MAPK gene families, which eventually suppresses escaspases $(22,26)$. There are two major families of serine/threonine protein kinases, the AGC family and the MAPK family. The AGC protein kinase family includes PDK1, cyclic AMP-dependent protein kinase, $\mathrm{PKB} / \mathrm{AKT}$ and $\mathrm{Ca}^{+}$-activated protein e kinase $(22,26)$. Among the DEGs, the present study found that PDK1, a key upstream gene of AKT, activates 23 protein kinases in the AGC family, including $\mathrm{PKB} / \mathrm{AKT}$, which is a key signaling intermediate involved in growth and 1 survival (27). Therefore, the levels of these genes were analyzed and it was found that the mRNA and protein expression levels of AKT and PDK1 were significantly increased in the Stra8-GC1 cells compared with those in the Control-GC1 cells. It has also been found that ANG2 increases cellular apoptosis by inhibiting the stimulation of AKT/Bcl-2 signaling $(28,29)$. OSGIN1 is a major mediator of cellular apoptosis under the control of tumor suppressor protein, p53 (19). In addition, BIRC6 silencing indirectly affects 
the regulators of p53 or directly binds and inhibits caspases (Casp3, 6, 7 and 9) through its BIR domain (30,31). Compared with the Control-GC1 cells, the present study found that Bcl-2 and BIRC6 were significantly increased; the mRNA levels of ANG2, p53 and OGSIN1 were decreased; and the protein levels of Bcl-2 in Stra8-GC1 cells was significantly increased. As these genes are all key factors in the AKT pathway, it was preliminarily hypothesized that the ability of Stra8 to inhibit apoptosis may be associated with the AKT pathway.

The other serine/threonine protein kinases family is the MAPK family, which includes RAF, ERK, MAPK kinase (MEK and MKK), MAPK kinase kinase, and JNK/p38 $(22,26)$. USP33, TCF4, and GSTP1 are MAPK signaling-related genes. In addition, USP33 is a gene involved in ERK signaling, which is important in the regulation of cellular proliferation, differentiation and $s$ apoptosis $(32,33)$. The phosphorylation of TCF4 at the serine/threonine proceeds via ERK and p-38 dependent pathways, and TCF4 knockdown has been shown to induce growth arrest and apoptosis in human colorectal cancer $(34,35)$. GSTP1, a dimeric protein, binds to JNK and suppresses downstream JNK g signaling (36). To the best of our knowledge, the JNK signaling pathway is involved in the processes of cell death and mediates stress-induced apoptosis via mitochondrial pathways with the release of cytochrome $c$-activating caspases (CASP3, -6 , and -7) $(36,37)$. Therefore, the present study detected the expression of P38, ERK, USP33, TCF4 and GSTP1. It was found that the mRNA levels of TCF4, USP33 and GSTP1 were significantly increased. In addition, the mRNA and protein expression levels of ERK were significantly increased in the Stra8-GC1 cells, compared with those in the Control-GC1 cells. As the MAPK pathway is an important AKT interaction pathway, it was further verified that Stra8 may have an anti-apoptotic effect through the AKT pathway.

Finally, the present study verified the expression of the caspase gene families and found that the expression of CASP3, one of the executioner caspases involved in cellular degradation during apoptosis, was decreased in the Stra8-GC1 cells compared with the Control-GC1 cells (38), further supporting the hypothesis that Stra8 functions to inhibit apoptosis. Therefore, a possible apoptotic pathway was characterized, in which Stra8 exerts an anti-apoptotic effect via the AKT signaling pathway. AKT directly or indirectly inactivates p53 by activating the $\mathrm{Bcl}-2$ family of genes and interacts with the MAPK family of genes, thereby inhibiting the caspase gene families and inducing an anti-apoptotic effect.

In the present study, the anti-apoptotic effects of Stra8 in the mouse male reproductive system were verified using in vitro and in vivo methods, and the possible involvement of the AKT signaling pathway was discussed; however, the investigations of potential Stra8-related signaling pathways were primarily based on gene expression analysis, which require further verification by investigations into the interaction between genes. Taken together, the findings of the present study provide a theoretical foundation for future in-depth functional investigations of Stra8.

\section{Acknowledgements}

Not applicable.

\section{Funding}

This study was supported by the National Natural Science Foundation of China (grant no. 31371174) and the Natural Science Foundation of Jiangsu Province, China (grant no. BK20131230).

\section{Availability of data and materials}

The datasets used and/or analyzed during the current study are available from the corresponding author on reasonable request.

\section{Authors' contributions}

XS, CN, JG designed and complemented the experiments; XS wrote the manuscript; MX, JX, and YH collected literatures and analyzed data; YZ designed the study and all authors read and approved the final version of the manuscript.

\section{Ethics approval and consent to participate}

All experiments were approved by the Committee on Animal Care of Yangzhou University.

\section{Patient consent for publication}

Not applicable.

\section{Competing interests}

The authors declare that they have no competing interests.

\section{References}

1. Tedesco M, La Sala G, Barbagallo F, De Felici M and Farini D: STRA8 shuttles between nucleus and cytoplasm and displays transcriptional activity. J Biol Chem 284: 35781-35793, 2009.

2. Ball RL, Fujiwara Y, Sun F, Hu J, Hibbs MA, Handel MA and Carter GW: Regulatory complexity revealed by integrated cytological and RNA-seq analyses of meiotic substages in mouse spermatocytes. BMC Genomics 17: 628, 2016.

3. Wang S, Wang X, Ma L, Lin X, Zhang D, Li Z, Wu Y, Zheng C, Feng X, Liao S, et al: Retinoic acid is sufficient for the in vitro induction of mouse spermatocytes. Stem Cell Reports 7: 80-94, 2016.

4. Koubova J, Hu YC, Bhattacharyya T, Soh YQ, Gill ME, Goodheart ML, Hogarth CA, Griswold MD and Page DC: Retinoic acid activates two pathways required for meiosis in mice. PLoS Genet 10: e1004541, 2014

5. Endo T, Romer KA, Anderson EL, Baltus AE, de Rooij DG and Page DC: Periodic retinoic acid-STRA8 signaling intersects with periodic germ-cell competencies to regulate spermatogenesis. Proc Natl Acad Sci USA 112: E2347-E2356, 2015.

6. Zhou Q, Nie R, Li Y, Friel P, Mitchell D, Hess RA, Small C and Griswold MD: Expression of stimulated by retinoic acid gene 8 (Stra8) in spermatogenic cells induced by retinoic acid: An in vivo study in vitamin A-sufficient postnatal murine testes. Biol Reprod 79: 35-42, 2008.

7. Mark M, Jacobs H, Oulad-Abdelghani M, Dennefeld C, Féret B, Vernet N, Codreanu CA, Chambon P and Ghyselinck NB. STRA8-deficient spermatocytes initiate, but fail to complete, meiosis and undergo premature chromosome condensation. J Cell Sci 121: 3233-3242, 2008.

8. Anderson EL, Baltus AE, Roepers-Gajadien HL, Hassold TJ, de Rooij DG, van Pelt AM and Page DC: Stra8 and its inducer, retinoic acid, regulate meiotic initiation in both spermatogenesis and oogenesis in mice. Proc Natl Acad Sci USA 105: 14976-14980, 2008. 
9. Zhang Y, Wang Y, Zuo Q, Li D, Zhang W, Wang F, Ji Y, Jin J, Lu Z, Wang M, et al: CRISPR/Cas9 mediated chicken Stra8 gene knockout and inhibition of male germ cell differentiation. PLoS One 12: e0172207, 2017.

10. Ma HT, Niu CM, Xia J, Shen XY, Xia MM, Hu YQ and Zhen Y: Stimulated by retinoic acid gene 8 (Stra8) plays important roles in many stages of spermatogenesis. Asian J Androl, 2018.

11. Livak KJ and Schmittgen TD: Analysis of relative gene expression data using real-time quantitative PCR and the 2- $\Delta \Delta C T$ method Methods 25: 402-408, 2001.

12. Miyamoto T, Sengoku K, Takuma N, Hasuike S, Hayashi H, Yamauchi T, Yamashita $\mathrm{T}$ and Ishikawa M: Isolation and expression analysis of the testis-specific gene, STRA8, stimulated by retinoic acid gene 8. J Assist Reprod Genet 19: 531-535, 2002.

13. Nair R and Shaha C: Diethylstilbestrol induces rat spermatogenic cell apoptosis in vivo through increased expression of spermatogenic cell Fas/FasL system. J Biol Chem 278: 6470-6481, 2003.

14. Liu T, Wang L, Chen H, Huang Y, Yang P, Ahmed N, Wang T, Liu Y and Chen Q: Molecular and cellular mechanisms of apoptosis during dissociated spermatogenesis. Front Physiol 8: 188, 2017.

15. Ning JZ, Rao T, Cheng F, Yu WM, Ruan Y, Yuan R, Zhu SM, Du Y and Xiao CC: Effect of varicocelectomy treatment on spermatogenesis and apoptosis via the induction of heat shock protein 70 in varicoceleinduced rats. Mol Med Rep 16: 5406-5412, 2017.

16. Li J, Chen F, Li C and Chen Y: Quinestrol induces spermatogenic apoptosis in vivo via increasing pro-apoptotic proteins in adult male mice. Tissue Cell 46: 318-325, 2014

17. Rogers R, Ouellet G, Brown C, Moyer B, Rasoulpour T and Hixon M: Cross-talk between the Akt and NF-kappaB signaling pathways inhibits MEHP-induced germ cell apoptosis. Toxicol Sci 106: 497-508, 2008

18. Abraham AG and O'Neill E: PI3K/Akt-mediated regulation of p53 in cancer. Biochem Soc Trans 42: 798-803, 2014.

19. Brennan MS, Matos MF, Richter KE, Li B and Scannevin RH: The NRF2 transcriptional target, OSGIN1, contributes to monomethyl fumarate-mediated cytoprotection in human astrocytes. Sci Rep 7: 42054, 2017.

20. Talos F, Petrenko O, Mena P and Moll UM: Mitochondrially targeted p53 has tumor suppressor activities in vivo. Cancer Res 65: 9971-9981, 2005.

21. Jia $\mathrm{X}, \mathrm{Xu} \mathrm{Y,} \mathrm{Wu} \mathrm{W,} \mathrm{Fan} \mathrm{Y,} \mathrm{Wang} \mathrm{G,} \mathrm{Zhang} \mathrm{T} \mathrm{and} \mathrm{Su} \mathrm{W:}$ Aroclor1254 disrupts the blood-testis barrier by promoting endocytosis and degradation of junction proteins via p38 MAPK pathway. Cell Death Dis 8: e2823, 2017.

22. Choudhury R, Bonacci T, Wang X, Truong A, Arceci A, Zhang Y, Mills CA, Kernan JL, Liu P and Emanuele MJ: The E3 ubiquitin Ligase SCF (Cyclin F) transmits AKT signaling to the cell-cycle machinery. Cell Rep 20: 3212-3222, 2017.

23. Matheny RW Jr and Adamo ML: Current perspectives on Akt Akt-ivation and Akt-ions. Exp Biol Med (Maywood) 234: 1264-1270, 2009.

24. Busada JT, Chappell VA, Niedenberger BA, Kaye EP, Keiper BD, Hogarth CA and Geyer CB: Retinoic acid regulates Kit translation during spermatogonial differentiation in the mouse. Dev Biol 397: 140-149, 2015.

25. Busada JT, Niedenberger BA, Velte EK, Keiper BD and Geyer CB: Mammalian target of rapamycin complex 1 (mTORC1) is required for mouse spermatogonial differentiation in vivo. Dev Biol 407: 90-102, 2015.
26. Harris TK.PDK1 and PKB/Akt: Ideal targets for development of new strategies to structure-based drug design. IUBMB Life 55 : $117-126,2003$.

27. Hurtado E, Cilleros V, Just L, Simó A, Nadal L, Tomàs M, Garcia N, Lanuza MA and Tomàs J: Synaptic activity and muscle contraction increases PDK1 and PKCbetaI phosphorylation in the presynaptic membrane of the neuromuscular junction. Front Mol Neurosci 10: 270, 2017.

28. Shen J, Frye M, Lee BL, Reinardy JL, McClung JM, Ding K, Kojima SSM, Xia H, Seidel C, Lima e Silva R, et al: Targeting VE-PTP activates TIE2 and stabilizes the ocular vasculature. J Clin Invest 124: 4564-4576, 2014.

29. Imanishi Y, Hu B, Xiao G, Yao X and Cheng SY: Angiopoietin-2, an angiogenic regulator, promotes initial growth and survival of breast cancer metastases to the lung through the integrin-linked kinase (ILK)-AKT-B cell lymphoma 2 (Bcl-2) pathway. J Biol Chem 286: 29249-29260, 2011

30. Ren J, Shi M, Liu R, Yang QH, Johnson T, Skarnes WC and Du C: The Birc6 (Bruce) gene regulates p53 and the mitochondrial pathway of apoptosis and is essential for mouse embryonic development. Proc Natl Acad Sci USA 102: 565-570, 2005.

31. Qiu XB and Goldberg AL: The membrane-associated inhibitor of apoptosis protein, BRUCE/Apollon, antagonizes both the precursor and mature forms of Smac and caspase-9. J Biol Chem 280: 174-182, 2005.

32. Wen P, Kong R, Liu J, Zhu L, Chen X, Li X, Nie Y, Wu K and Wu JY: USP33, a new player in lung cancer, mediates Slit-Robo signaling. Protein Cell 5: 704-713, 2014.

33. Liu H, Zhang Q, Li K, Gong Z, Liu Z, Xu Y, Swaney MH, Xiao K and Chen Y: Prognostic significance of USP33 in advanced colorectal cancer patients: New insights into $\beta$-arrestin-dependent ERK signaling. Oncotarget 7: 81223-81240, 2016.

34. Yu N, Song Z, Zhang $\mathrm{K}$ and Yang X: MAD2B acts as a negative regulatory partner of TCF4 on proliferation in human dermal papilla cells. Sci Rep 7: 11687, 2017.

35. Jeong JB, Lee J, and Lee SH: TCF4 is a molecular target of resveratrol in the prevention of colorectal cancer. Int J Mol Sci 16: $10411-10425,2015$.

36. Domazetovic V, Fontani F, Marcucci G, Iantomasi T, Brandi ML and Vincenzini MT: Estrogen inhibits starvation-induced apoptosis in osteocytes by a redox-independent process involving association of JNK and glutathione S-transferase P1-1. FEBS Open Bio 7: 705-718, 2017.

37. Okamura T, Antoun G, Keir ST, Friedman H, Bigner DD and Ali-Osman F: Phosphorylation of glutathione S-transferase P1 (GSTP1) by epidermal growth factor receptor (EGFR) promotes formation of the GSTP1-c-Jun N-terminal kinase (JNK) complex and suppresses JNK downstream signaling and apoptosis in brain tumor cells. J Biol Chem 290: 30866-30878, 2015.

38. Flanagan L, Meyer M, Fay J, Curry S, Bacon O, Duessmann H, John K, Boland KC, McNamara DA, Kay EW, et al: Low levels of Caspase-3 predict favourable response to 5FU-based chemotherapy in advanced colorectal cancer: Caspase-3 inhibition as a therapeutic approach. Cell Death Dis 7: e2087, 2016. 